\title{
Spontaneous Regression of Hepatocellular Carcinoma after Eradication of HCV
}

\author{
Eric F. Martin ${ }^{1}$, Michael Huang ${ }^{2}$, Beatrice Madrazo ${ }^{3}$ and Cynthia Levy ${ }^{1 *}$ \\ ${ }^{1}$ Division of Hepatology, University of Miami Miller School of Medicine, Miami, Florida, USA \\ ${ }^{2}$ Division of Gastroenterology, University of Miami Miller School of Medicine, Miami, Florida, USA \\ ${ }^{3}$ Division of Radiology, University of Miami Miller School of Medicine, Miami, Florida, USA
}

Received: September 10, 2014; Accepted: November 12, 2014; Published: December 02, 2014

*Corresponding author: Cynthia Levy, University of Miami Miller School of Medicine, Division of Hepatology, 1500 NW 12th Ave, Suite 1101, Miami, FL 33136, USA, Tel: 305-243-2330; E-mail: clevy@med.miami.edu

\begin{abstract}
Spontaneous regression of Hepatocellular Carcinoma (HCC) is uncommon. Several factors have been suggested as the mechanisms behind spontaneous regression of HCC, namely reduction in blood supply, inflammation, and immunological factors; however, the precise pathogenesis is unknown. We present a case of spontaneous regression of HCC in a 57 year old female with cirrhosis secondary to chronic Hepatitis C Virus (HCV). On a triple phase CT scan of the liver, she was found to have a $26 \mathrm{~mm}$ liver lesion fulfilling Liver Imaging Reporting and Data Systems (LI-RADs) 5 criteria for HCC with elevated serum Alpha Fetoprotein (AFP) level to $218 \mathrm{ng} / \mathrm{mL}$. She was started on sofosbuvir and simeprevir for treatment of HCV, genotype 1b. HCV RNA was undetectable by week four of treatment and she successfully completed 12 weeks of therapy. Repeat triple phase CT scan of the liver one month after completing anti-HCV therapy, aforementioned liver lesion reduced to $6 \mathrm{~mm} \times 5 \mathrm{~mm}$. Serum AFP also decreased from $218 \mathrm{ng} / \mathrm{mL}$ before treatment to $37 \mathrm{ng} / \mathrm{mL}$ during treatment and to $22 \mathrm{ng} / \mathrm{mL}$ after treatment. Spontaneous regression of HCC with anti-HCV therapy and successful HCV eradication is new and previously unexplained
\end{abstract}

Keywords: Spontaneous regression; Hepatocellular carcinoma; Hepatitis C virus

\section{Introduction}

Hepatocellular Carcinoma (HCC) is the most common cause of primary liver cancer and the fifth most common cancer worldwide. Although early detection and development of therapies for HCC often improve prognosis, the prognosis of untreated, advanced HCC remains poor with a median survival of less than six months. Spontaneous regression of HCC is rare with a reported incidence rate of 1 in 140,000 cases of HCC [1]. HCC regression is generally defined as the partial or complete regression of HCC in the absence of specific anti-neoplastic therapy, namely chemotherapy and/or locoregional therapy. The underlying mechanism of HCC regression, however, remains unknown. HCC regression following antiviral therapy for chronic HCV, specifically with sofosbuvir, has not been previously reported. Further understanding of this phenomenon could lead to novel strategies for HCC treatment. We report here one case of spontaneous regression of HCC following treatment of chronic HCV with sofosbuvir and simeprevir.

\section{Case Report}

A 57 year old black female with Child-Pugh A cirrhosis secondary to chronic HCV, genotype $1 b$, was seen in the Hepatology clinic. She is a non-responder to a previous 6-month treatment course of Pegylated interferon- $2 \alpha$ and Ribavirin. Previous surveillance abdominal imaging, which included ultrasonography, triple phase CT scan of the liver, and MRI abdomen with and without contrast, showed no evidence of HCC. The last imaging was an MRI of the abdomen with and without contrast in July 2012 that showed no evidence of hepatocellular carcinoma, but did reveal multiple small intrahepatic lesions with delayed contrast pooling suggestive of regenerative nodules. The serum AFP level at this time was elevated at $218 \mathrm{ng} / \mathrm{mL}$, which was previously $71 \mathrm{ng} / \mathrm{mL}$. Follow-up triple phase CT scan of the liver in January 2014 revealed a $26 \mathrm{~mm}$ x $18 \mathrm{~mm}$ lesion in segment VII fulfilling LI-RADS 5 criteria, which was highly suggestive of HCC. AFP at that time was $196 \mathrm{ng} / \mathrm{mL}$. She was referred to the liver transplant clinic where she was started on anti-HCV treatment with sofosbuvir $400 \mathrm{mg} /$ day and simeprevir 150mg/ day with recommendations to proceed with an evaluation for liver transplantation. When treatment was initiated in February 2014, her HCV RNA was 1 million IU/mL. By week 4, HCV RNA became undetectable and remained so throughout the 12-week course of sofosbuvir and simeprevir, which she completed in May 2014.

Of note, during the transplant evaluation she was diagnosed with latent tuberculosis by positive QuantiFERON-TB Gold test and negative chest X-ray. In May 2014, she was started on isoniazid $900 \mathrm{mg} /$ day for an anticipated nine months. Repeat triple phase CT scan of the liver in April 2014 demonstrated the lesion measured $13 \mathrm{~mm}$ (compared to $26 \mathrm{~mm} 3$ months earlier). Her AFP also decreased from $196 \mathrm{ng} / \mathrm{mL}$ to $37 \mathrm{ng} / \mathrm{mL}$. Finally, follow-up triple phase liver CT scan in July 2014 showed that the lesion in segment VII had reduced to mere $6 \mathrm{~mm} \times 5 \mathrm{~mm}$ with an AFP of $22 \mathrm{ng} / \mathrm{mL}$. Figure 1 demonstrates the regression of HCC 
in this patient before starting sofosbuvir and simeprevir (Figure 1A-C) and after finishing sofosbuvir and simeprevir (Figure 1DF). Table 1 shows the trend of HCV RNA, AFP, and the size of the liver mass at initial visit, during HCV treatment, and at last visit.

\section{Discussion}

Spontaneous regression of HCC is a rare happening, yet the true incidence is difficult to accurately define. Upon reviewing 10 randomized controlled trials including 1640 patients with HCC, Oquiñena et al. [2] estimated the incidence of spontaneous regression of HCC to be $0.4 \%$. The number of cases of spontaneous regression of HCC reported in the literature is higher in HCC than in other malignancies, which likely reflects a higher incidence of HCC compared to other malignancies. Although a large majority of the previously reported cases involves partial regression, few cases describe complete regression of HCC [3]. There are even case reports of complete spontaneous regression of HCC with metastasis to the chest wall [4], portal vein tumor thrombus [5], and pulmonary metastases [6]. In the event of the portal vein tumor thrombus and pulmonary metastases, both patients experienced chronic HCV. To our knowledge, no previous cases of HCC regression coinciding with anti-HCV therapy have been reported.

No evidence exists to support a correlation between the underlying etiology of liver disease and spontaneous regression of HCC. One of the largest and most recent reviews of the literature by Oquiñena et al. [7] included 59 clinical reports of spontaneous regression of HCC. The clinical profile and demographics varied widely in their cohort of patients. A plausible explanation for the pathogenic mechanism underlying HCC regression was offered in 27 of the 59 cases (46\%). Of those identified, ischemia due to hemorrhagic shock or hepatic artery thrombosis was the most commonly detected cause, occurring in 10 of 59 cases (17\%). Several other factors, namely inflammation, immunological factors, and reduction in blood supply leading to tumor hypoxia have also been suggested as likely mechanisms behind spontaneous regression of HCC, but the precise pathogenesis

Table 1: The trend of HCV RNA, AFP, and the size of the liver mass at initial visit, during HCV treatment and at last visit.

\begin{tabular}{|c|c|c|c|c|c|c|}
\hline \multirow[b]{2}{*}{ Date } & \multicolumn{2}{|c|}{ Prior to treatment } & \multicolumn{3}{|c|}{ During treatment } & \multirow{2}{*}{$\begin{array}{c}\text { After treatment } \\
\text { July } \\
2014\end{array}$} \\
\hline & March 2013 & January 2014 & $\begin{array}{c}\text { February } \\
2014\end{array}$ & $\begin{array}{l}\text { April } \\
2014\end{array}$ & $\begin{array}{l}\text { May } \\
2014\end{array}$ & \\
\hline HCV RNA (IU/mL) & 1 million & 1 million & \multirow{3}{*}{$\begin{array}{l}\text { Started sofosbuvir } \\
\text { and simeprevir }\end{array}$} & undetectable & \multirow{3}{*}{$\begin{array}{l}\text { Completed } \\
\text { sofosbuvir and } \\
\text { simeprevir }\end{array}$} & undetectable \\
\hline $\mathrm{AFP}(\mathrm{ng} / \mathrm{mL})$ & 71 & 196 & & 37 & & 22 \\
\hline Size of liver mass & no HCC seen & $26 \mathrm{~mm} \times 18 \mathrm{~mm}$ & & $13 \mathrm{~mm} \times 13 \mathrm{~mm}$ & & $6 \mathrm{~mm} \times 5 \mathrm{~mm}$ \\
\hline
\end{tabular}
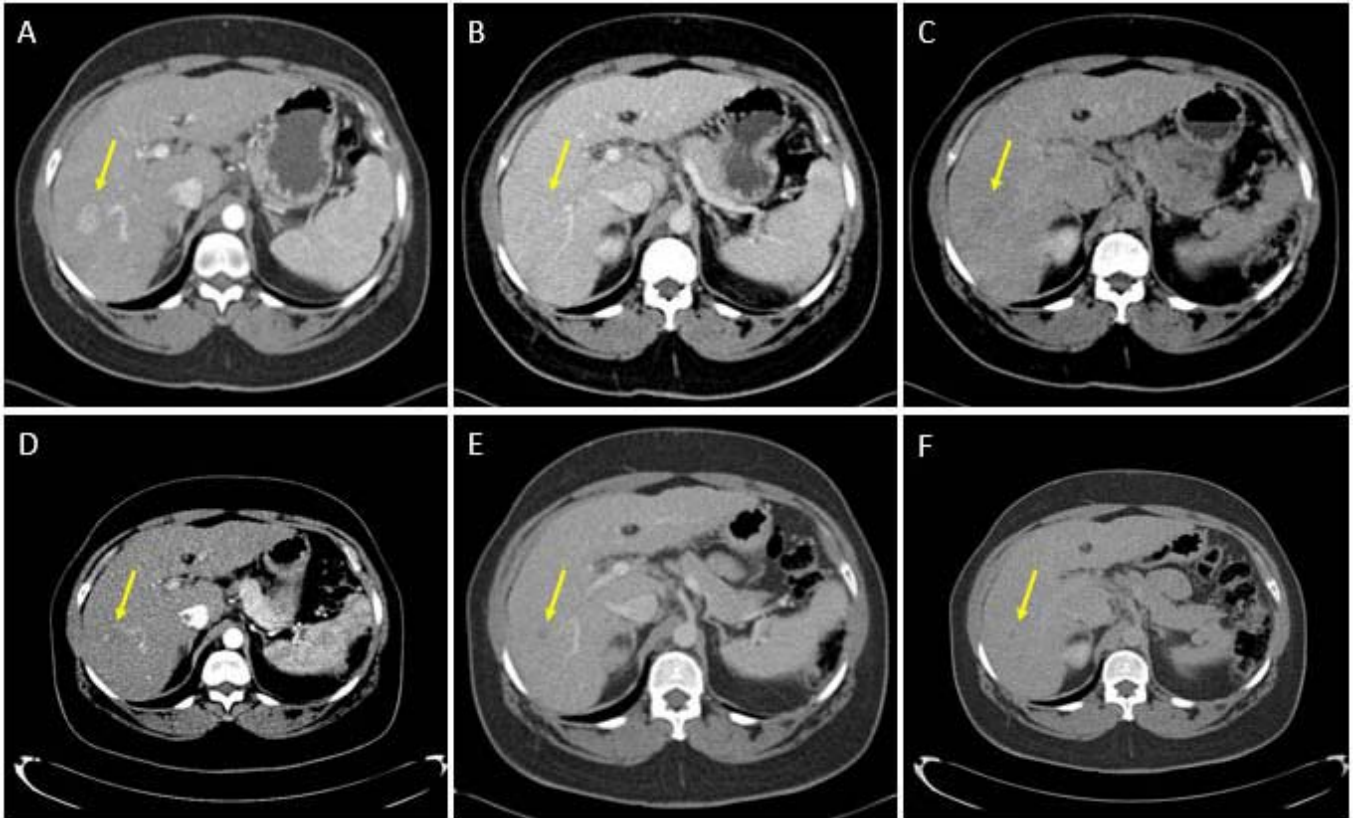

Figure 1: Representative images from triple phase CT scan of the liver before starting sofosbuvir and simeprevir (A-C) and after finishing sofosbuvir and simeprevir (D-F). 1A. Arterial phase axial image demonstrates a $17 \mathrm{~mm}$ x $23 \mathrm{~mm}$ hyperenhancing lesion seen in segment VII (arrow) before starting sofosbuvir. 1B. Portovenous phase axial image demonstrates faint perception of lesion's capsule (arrow). 1C. Delayed phase axial image reveals wash-out and capsular perception (arrow). 1D. Arterial phase axial image now demonstrates a $6 \mathrm{~mm} \times 5 \mathrm{~mm}$ lesion, which has significantly decreased in size from the previous $26 \mathrm{~mm} \times 18 \mathrm{~mm}$ lesion seen 7 months earlier (Figure $1 \mathrm{~A}$ ). A barely perceptible $5 \mathrm{~mm}$ low attenuation lesion is seen on portovenous phase (1E) and delayed phase (1F). 
is unknown $[3,8]$. In a more recent systematic review of 75 patients with spontaneous remission of HCC, the cause of regression was attributed to tumor hypoxia in $21(28 \%)$ and systemic inflammatory response in $25(33 \%)$ patients while the cause of HCC regression of the remaining 29 (38\%) patients were unknown [9]. Recognized causes of tissue hypoxia include spontaneous hepatic artery thrombosis, an occlusive portal vein thrombosis, development of a large arterioportal shunt, angiospasm during angiography, and prolonged hypotension due to massive gastrointestinal bleeding. Tumor hypoxia as a mechanism for HCC regression seems intuitive as it is the basis of established treatment options for HCC, namely Transhepatic Arterial Chemoembolization (TACE) and the oral multiple kinase inhibitor sorafenib. TACE occludes the arterial supply to the tumor, which effectively reproduces the effect of HCC regression that has been reported after spontaneous arterial thrombosis [1012]. Likewise, sorafenib relies upon induction of tumor hypoxia due to its anti-angiogenesis effect. It is possible that the presence of an arterioportal shunt near the liver tumor may alter the dynamics of blood flow to the tumor, which is essential for tumor growth. Similar to TACE, tumor infarction due to disruption of the feeding vessel due to subintimal injury and tumor invasion could produce tumor hypoxia leading to tissue necrosis and regression of HCC. Rapid tumor growth, administration of vitamin K, abstinence from alcohol, consumption of herbal medicines, high fevers, and gastrointestinal bleeding have also been suggested as factors causing spontaneous regression of HCC [13]. Of note, our patient had no radiographic evidence of an arterioportal shunt or spontaneous hepatic artery or portal vein thrombosis.

Perhaps most compelling are the immunological factors that have been implicated in the spontaneous regression of HCC. For over three decades, it has been proposed that the most important factor in spontaneous regression of cancer is stimulation of the immune process [14]. It was previously shown that patients with HCC, particularly those with chronic HCV, had significant impairment in T-cell responses at baseline, which correlated with tumor burden and poor outcome [15]. A previous report demonstrated that treatment with activated T-lymphocytes after surgery reduced the recurrence rates of HCC, suggesting that HCC regression may be associated with the host immune response [16]. In a cohort of liver transplant recipients, those who achieved eradication of HCV after antiviral therapy achieved restoration of HCV-specific T-cell responses. On the other hand, patients with progressive HCV recurrence after liver transplant that failed antiviral therapy demonstrated declining frequencies of HCVspecific T-cells [17]. Similarly, in a cohort of coinfected patients with HCV and HIV who were treated with Highly-Active AntiRetroviral Therapy (HAART), HCV Ab-reactivity was associated with an inferior virologic response to HAART compared to HCV Ab-negative patients [18]. It is possible that HCV lowers CD4+ T-cell recovery through direct pathogenic effect on the lymphocytes. It has also been hypothesized that CD4+ T-cell depletion results from ongoing cell activation and apoptosis drive by HCV [19]. The studies present data that strongly support the theory of immune reconstitution following eradication of HCV, which may represent the immunologic trigger associated with spontaneous regression of HCC.

\section{Conclusion}

Spontaneous regression of HCC is rarely reported. Its coincidental course with anti-HCV therapy and HCV eradication, however, is new and yet unexplained. Further observational data and analysis of patients with spontaneous regression of HCC are required to build a clinical significance and potentially guide future growth of novel treatment strategies for HCC.

\section{References}

1. Chang WY. Complete spontaneous regression of cancer: four case reports, review of literature, and discussion of possible mechanisms involved. Hawaii Med J. 2000; 59(10): 379-387.

2. Oquiñena S, Guillen-Grima F, Inarrairaegui M, Zozaya JM, Sangro B. Spontaneous regression of hepatocellular carcinoma: a systemic review. Eur J Gastroenterol Hepatol. 2009; 21(3): 254-257. doi: 10.1097/MEG.0b013e328324b6a2.

3. Meza-Junco J, Montaño-Loza AJ, Martinez-Benítez B, CabreraAleksandrova T. Spontaneous partial regression of hepatocellular carcinoma in a cirrhotic patient. Ann Hepatol. 2007; 6(1): 66-69.

4. Jeson SW, Lee MK, Lee YD, Seo HE, Cho CM, Tak WY, et al. Clear cell hepatocellular carcinoma with spontaneous regression of primary and metastatic lesions. Korean J Intern Med. 2005; 20(3): 268-273.

5. Liai T, Sato Y, Nabatame N, Yamamoto S, Makino S, Hatakeyama K. Spontaneous complete regression of hepatocellular carcinoma with portal vein tumor thrombus. Hepatogastroenterology. 2003; 50(53): 1628-1630.

6. Toyoda H, Sugimura S, Fukuda K, Mabuchi T. Hepatocellular carcinoma with spontaneous regression of multiple lung metastases. Pathol Int. 1999; 49(10): 893-897.

7. Oquiñena S, Inarrairaegui M, Vila JJ, Alegre F, Zozaya JM, Sangro B. Spontaneous Regression of Hepatocellular Carcinoma: Three Case Reports and a Categorized Review of the Literature. Dig Dis Sic. 2009; 54(5): 1147-1153. doi: 10.1007/s10620-008-0447-z.

8. Blondon H, Fritsch L, Cherqui D. Two cases of spontaneous regression of multicentric hepatocellular carcinoma after intraperitoneal rupture: possible role of immune mechanisms. Eur J Gastroenterol Hepatol. 2004; 16(12): 1355-1359.

9. Huz JI, Melis M, Sarpel U. Spontaneous regression of hepatocellular carcinoma is most often associated with tumour hypoxia or a systemic inflammatory response. HPB (Oxford). 2012; 14(8): 500-505. doi: 10.1111/j.1477-2574.2012.00478.x.

10. Yano Y, Yamashita F, Kuwaki K, Fukumori K, Kato O, Kiyomatsu K, et al. Partial spontaneous regression of hepatocellular carcinoma: a case with high concentrations of serum lens culinaris agglutinin-reactive alpha fetoprotein. Kurume Med J. 2005; 52(3): 97-103.

11. Ohtani H, Yamazaki O, Matsuyama M, Horii K, Shimizu S, Oka H. Spontaneous regression of hepatocellular carcinoma: report of a case. Surg Today. 2005; 35(12): 1081-1086.

12. Imaoka S, Sasaki Y, Masutani S, Ishikawa O, Furukawa $\mathrm{H}$, Kabuto T, et al. Necrosis of hepatocellular carcinoma caused by spontaneously arising arterial thrombus. Hepatogastroenterology. 1994; 41(4): 359362 .

13. Nouso K, Uematsu S, Shiraga K, Okamoto R, Harada R, Takayama $\mathrm{S}$, et al. Regression of hepatocellular carcinoma during vitamin $\mathrm{K}$ administration. World J Gastroenterol. 2005; 11(42): 6722-6724. 
14. Cole WH. Efforts to explain spontaneous regression of cancer. J Surg Oncol. 1981; 17(3): 201-209.

15. Cabrera R, Ararat M, Cao M, Xu Y, Wasserfall C, Atkinson MA, et al Hepatocellular carcinoma immunopathogenesis: clinical evidence for global $\mathrm{T}$ cell defects and an immunomodulatory role for soluble CD25 (sCD25). Dig Dis Sci. 2010; 55(2): 484-495. doi: 10.1007/s10620-0090955-5.

16. Anthony PP. Hepatocellular carcinoma: an overview. Histopathology. 2001; 39(2): 109-118.

17. Weston SJ, Leistikow RL, Reddy KR, Torres M, Wertheimer AM, Lewinsohn DM, et al. Reconstitution of Hepatitis C Virus-Specific
T-cell-Mediated Immunity after Liver Transplantation. Hepatology. 2005; 41(1): 72-81.

18. Motta D, Brianese N, Foca E, Nasta P, Maggiolo F, Fabbiani M, et al. Virological effectiveness and CD4+ T-cell increase over early and late courses in HIV infected patients on antiretroviral therapy: focus on HCV and anchor class received. AIDS Res Ther. 2012; 9(1): 18. doi: 10.1186/1742-6405-9-18.

19. Nunez M, Soriano V, Lopez M, Ballesteros C, Cascajero A, GonzálezLahoz J, et al. Coinfection with hepatitis $C$ increases lymphocyte apoptosis in HIV-infected patients. Clin Infect Dis. 2006; 43(9): 12091212. 